\title{
ISOMER RATIOS IN PHOTONUCLEAR REACTIONS WITH MULTIPLE NEUTRON EMISSION
}

\author{
O.M. Vodin $^{1 *}$, O.A.Bezshyyko ${ }^{2}$, L. O. Golinka-Bezshyyko ${ }^{2}$, I. M. Kadenko ${ }^{2}$, \\ V.A.Kushnir ${ }^{1}$, A.V.Kotenko ${ }^{2}$, O.V.Lubynets ${ }^{2}$, V.V. Mitrochenko ${ }^{1}$, \\ S.M. Olejnik ${ }^{1}$, S.A.Perezhogin ${ }^{1}$, C. Vallerand ${ }^{3}$ \\ ${ }^{1}$ National Science Center "Kharkiv Institute of Physics and Technology", 61108 Kharkiv, Ukraine; \\ ${ }^{2}$ Taras Shevchenko National University of Kyiv, 01601, Kyiv, Ukraine; \\ ${ }^{3}$ Laboratoire de l'Acce'le'rateur Line'aire, 91898 Orsay, France (LAL)
}

(Received August 2, 2018)

\begin{abstract}
The aim of this work is studying of gamma-quanta energy dependence of isomeric ratios in $A(\gamma, x n)^{m, g}(A-x n)$ reactions in energy range $35 \ldots 100 \mathrm{MeV}$, i.e. beyond Great Dipole Resonance (GDR) region. Experimental data were taken from international database EXFOR for a wide range of nuclear mases $(55<A<181)$. Theoretical values of isomeric ratios were obtained using TALYS-1.8 code. Several models of level densities with both enabled and disabled pre-equilibrium mechanism were considered in our simulations. Obtained results let us to make conclusions about different mechanisms of photonuclear reactions on certain nuclei, energy dependence of their relative contribution.
\end{abstract}

PACS: 25.20, 13.60.H

\section{INTRODUCTION}

Nuclear reactions with photons in the input channel have some advantages which are used for investigation of general physical dependencies and some special features of photonuclear reactions. First of all, gamma-quanta do not contribute large angular momentum to the nuclei, and excitation energy of nuclei does not include binding energy of the incident particle. Characteristics of photonuclear reactions are well studied in the energy region of giant dipole resonance and at energies higher than pion production threshold. Energies between GDR and threshold of pion production $(30 \ldots 100 \mathrm{MeV})$ are studied not so well from both experimental and theoretical point of view. It is related with small values of cross sections in this energy region, lack of gamma-quanta sources with fine energy regulation and some historical reasons [1].

There are two main models of nuclear reactions mechanisms: compound nucleus model and direct reaction model. The main idea of compound nuclei model is that energy of incident particle is uniformly distributed between all the nucleons, and nucleon emission is considered as evaporation process. This model is also called statistical model. There is another mechanism considering particle emission before statistical equilibrium is established. The nucleon can be knocked out with the incident particle; this process is called direct reaction. It is clear that increasing of number of interactions in the nucleus (increasing ofreaction time) leads to reduction of the connection between input and output channels and increasing the contribution of statistical processes.
There are several program codes for nuclear reactions description. One of them is TALYS [1], which has open source. It can be used for evaluation of cross sections, isomeric ratios and other characteristics of nuclear reactions with neutrons, protons, photons, deuterons, ${ }^{3} \mathrm{H},{ }^{3} \mathrm{He}$ and $\alpha$-particles in the input channel.

In the TALYS code modern model approaches for description of direct reactions, pre-equilibrium processes, reactions with compound nuclei production and fission processes are implemented. Reaction mechanisms are considered in wide ranges of incident particle energies $\left(10^{-11} \mathrm{MeV}<E<1000 \mathrm{GeV}\right)$ and target nuclei masses $(5<A<339)$. An important advantage of TALYS code is automatic use of model parameters and estimated nuclear data from RIPL-3 [2] library[4].

TALYS provides 6 model approaches (LD1-LD6) for level density evaluation: 3 phenomenological models and 3 level density sets derived from microscopic models.

LD1: Constant temperature + Fermi gas model. In this model introduced by Gilbert and Cameron [3], the excitation energy range is divided into a low energy part from $E_{0}$ up to a matching energy $E_{M}$, where the so-called constant temperature law applies and a high energy part above, where the Fermi gas model applies. Hence, for the total level density we have

$$
\begin{array}{ll}
\rho^{t o t}\left(E_{x}\right)=\rho_{F}^{t o t}\left(E_{x}\right), & \text { if } \quad E_{x}>E_{M}, \\
\rho^{t o t}\left(E_{x}\right)=\rho_{T}^{t o t}\left(E_{x}\right), & \text { if } \quad E_{x}<E_{M} .
\end{array}
$$

LD2: Back-shifted Fermi gas model In the Back-

\footnotetext{
*Corresponding author E-mail address: vodin@kipt.kharkov.ua
} 
shifted Fermi gas Model (BFM) [4], the pairing energy is treated as an adjustable parameter and the Fermi gas expression is used all the way down to $E_{0}$.

LD3: Generalised superfluid model (GSM). Model takes superconductive pairing correlations into account according to the Bardeen-Cooper-Schrieffer theory. The phenomenological version of the model $[7,8]$ is characterized by a phase transition from a superfluid behavior at low energy, where pairing correlations strongly influence the level density, to a high energy region which is described by the Fermi gas model. The GSM thus resembles the constant temperature model to the extent that it distinguishes between a low energy and a high energy region, although for the GSM this distinction follows naturally from the theory and does not depend on specific discrete levels that determine a matching energy. Instead, the model automatically provides a constant temperature-like behavior at low energies.

LD4: Microscopic level densities (Skyrme force) from Goriely's tables Using this model allows to read tables of microscopic level densities from RIPL database. These table were computed by $S$. Goriely on the basis of Hartree-Fock calculations for excitation energies up to $150 \mathrm{MeV}$ and for spin values up to $I=30$.

LD5: Microscopic level densities (Skyrme force) from Hilaire's combinatorial tables The combinatorial model includes a detailed microscopic calculation of the intrinsic state density and collective enhancement. The only phenomenological aspect of the model is a simple damping function for the transition from spherical to deformed.

LD6: Microscopic level densities (temperature dependent HFB, Gogny force) from Hilaire's combinatorial tables.

We consider reactions both with enabled and disabled pre-equilibrium mechanism. Pre-equilibrium mechanism of nuclear reactions is an intermediate type between direct reaction and reaction via compound nuclei. Pre-equilibrium emission takes place after the first stage of the reaction but long before statistical equilibrium of the compound nucleus is attained. The pre-equilibrium contribution becomes only sizable for incident energies several $\mathrm{MeV}$ higher than the excitation energy of the last discrete level of the target nucleus.

\section{EXPERIMENTAL METHOD}

The product nuclei of photonuclear reaction can be both in ground and metastable (also called isomeric) state. Photonuclear reaction is characterized with isomeric ratio. In case of monochromatic incident particles flow the isomeric ratio can be determined as cross sections ratio $\sigma_{m}(E) / \sigma_{g}(E)$, where $\sigma_{g(m)}(E)$ is the ground (isomeric) nuclei state production cross section. Also isomeric ratio can be defined as $\sigma_{H}(E) / \sigma_{L}(E)$, i.e. the ratio between cross sections of states with high and low spin.

If the incident particles spectrum is substantially different from monoenergetic (especially in experi- ments with bremsstrahlung $\gamma$-quants) the isomeric ratio of yields is used:

$$
d\left(E_{\max }\right)=\frac{Y_{m}}{Y_{g}},
$$

where $Y_{g(m)}$ is the ground (isomeric) state yield for the maximum bremsstrahlung energy $E_{\max }$.

The reaction yield is determined as

$$
Y_{m, g}=N_{t} \int_{E_{t h}^{m, g}}^{E_{\max }} \sigma_{m, g}(E) W\left(E, E_{\max }\right) d E,
$$

where:

$N_{t}$ is the number of target nuclei;

$E_{\max }$ - maximum energy of incident particles;

$\sigma_{m, g}(E)$ - ground (isomeric) nuclei state production cross section;

$W\left(E, E_{\max }\right)$ - energy spectrum of incident particles; $E_{t h}^{m, g}$ - threshold energy of ground (isomeric) state nuclei production.

Energy spectrum depends on $\gamma$-quantum production cross section. It is clear, because $W\left(E_{\gamma}, E_{\max }\right)$ is energy distribution of particles:

$$
W\left(E_{\gamma}, E_{\max }\right)=\frac{d N}{d E},
$$

where $N$ is the number of $\gamma$-quanta emitted from bremsstrahlung target. The number of $\gamma$-quanta emitted from bremsstrahlung target into solid angle $d \Omega$ is

$$
d N=N_{0} \frac{n}{S} \frac{d \sigma}{d \Omega} d \Omega
$$

where:

$N_{0}$ is the number of electrons interacting with bremsstrahlung target;

$n$-number of bremsstrahlung target nuclei with cross section $S$.

The expression for the flux of $\gamma$-quanta with energies in the range $\left(E_{\gamma} \ldots E_{\gamma}+d E_{\gamma}\right)$ emitted into the solid angle $d \Omega$ can be written in the following form:

$$
d W\left(E_{\gamma}, E_{\max }\right)=N_{0} \frac{n}{S} \frac{d^{2} \sigma}{d \Omega d E_{\gamma}} d \Omega .
$$

Integrating (5) over the solid angle we have

$$
W\left(E_{\gamma}, E_{\max }\right)=\int_{\Omega} N_{0} \frac{n}{S} \frac{d^{2} \sigma}{d \Omega d E_{\gamma}} d \Omega .
$$

Assuming the target to be thin (twice thinner than radiation length of material [7]), we use the bremsstrahlung energy spectrum calculated in work [8]. The cross section of bremsstrahlung $\gamma$-quanta production is called Shiff spectrum [9]:

$$
\begin{gathered}
\frac{d \sigma}{d \Omega}=\frac{1}{2 \pi} \frac{4 Z^{2}}{137} r_{0}^{2}\left(\frac{E_{0}}{m c^{2}}\right)^{2} \frac{d E_{\gamma}}{E_{\gamma}} \times \\
{\left[\frac{E_{0}^{2}+E^{2}}{E_{0}^{2}} \ln M(0)-\frac{\left(E_{0}+E\right)^{2}}{E_{0}^{2}}\right],}
\end{gathered}
$$

where:

$E$ is the total energy of the scattered electron; 


\section{ANALYSIS}

$Z$ - atom number of target material;

$r_{0}$ - classical electron radius;

$$
\frac{1}{M(0)}=\left(\frac{m c^{2} E_{\gamma}}{2 E_{0} E}\right)^{2}+\frac{Z^{2 / 3}}{111^{2}}
$$

In Shiff's approximation $d^{2} \sigma / d \Omega d E_{\gamma}$ is assumed not to have angle dependence, and $\gamma$-qaunta emission is limited with solid angle $\Omega_{0}$. Then energy spectrum $W\left(E_{\gamma}, E_{\max }\right)$ is proportional to $d^{2} \sigma / d \Omega d E_{\gamma}$ with proportional coefficient $N_{0}(n / s) \Omega_{0}$.

Considering (3)-(6) we obtain the expression for isomeric ratio:

$$
D\left(E_{\max }\right)=\frac{Y_{H}}{Y_{L}}=\frac{\int_{E_{t h}^{H}}^{E_{\max }} \sigma_{H}\left(E_{\gamma}\right) \frac{d^{2} \sigma}{d \Omega d E_{\gamma}} d E_{\gamma}}{\int_{E_{\text {th }}^{L}}^{E_{\max }} \sigma_{L}\left(E_{\gamma}\right) \frac{d^{2} \sigma}{d \Omega d E_{\gamma}} d E_{\gamma}} .
$$

Published data of isomeric ratios is represented in a variety of forms: $Y_{m} / Y_{g}, Y_{m} /\left(Y_{g}+Y_{m}\right), Y_{H} / Y_{L}$, $\sigma_{m} / \sigma_{g}, \sigma_{m} /\left(\sigma_{g}+\sigma_{m}\right), \sigma_{H} / \sigma_{L}$. To simplify comparison isomeric ratios of different authors are recalculated and represented in Table in $Y_{H} / Y_{L}$ form. In the first column of Table there is a photonuclear reaction, its threshold $\left(E_{t h}\right)$, spin and parity of target, ground and isomeric nuclei $(t, g$ and $m$ respectively). In the second column there is maximum energy of bremsstrahlung photons. In the third column there is an experimental value of isomeric ratio $D=Y_{H} / Y_{L}$. The fourth column contains reference to the experimental data represented in the third column; in the 5 -th and 6 -th column there are theoretically predicted values of isomeric ratio separated with slashes (from 1-st (LD1) to 6-th (LD6) level density model, both with enabled and disabled pre-equilibrium mechanism).

\begin{tabular}{|c|c|c|c|c|c|}
\hline \multirow[t]{2}{*}{ Reaction } & \multirow{2}{*}{$\begin{array}{l}E_{\max }, \\
\mathrm{MeV}\end{array}$} & \multirow[t]{2}{*}{$D$} & \multirow[t]{2}{*}{ Ref. } & \multicolumn{2}{|c|}{ Theoretical value of $D$} \\
\hline & & & & disabled pre-equilibrium & enabled pre-equilibrium \\
\hline $\begin{array}{l}{ }^{55} \mathrm{Mn}(\gamma, 3 n)^{52} \mathrm{Mn} \\
E_{t h}=31.2 \mathrm{MeV} \\
\mathrm{t}: 5 / 2^{-} \\
\mathrm{g}: 6^{+} \\
\mathrm{m}: 2^{+}\end{array}$ & $\begin{array}{r}49 \\
100\end{array}$ & $\begin{array}{l}0.41 \pm 0.02 \\
0.77 \pm 0.06\end{array}$ & $\begin{array}{l}{[10]} \\
{[11]}\end{array}$ & $\begin{array}{l}0.45 / 0.50 / 0.50 / 0.55 / 0.56 / 0.64 \\
0.46 / 0.53 / 0.53 / 0.60 / 0.64 / 0.73\end{array}$ & $\begin{array}{l}0.48 / 0.53 / 0.53 / 0.59 / 0.59 / 0.67 \\
0.58 / 0.65 / 0.64 / 0.74 / 0.75 / 0.83\end{array}$ \\
\hline $\begin{array}{l}{ }^{88} \mathrm{Sr}(\gamma, 3 n)^{85} \mathrm{Sr} \\
E_{t h}=31.3 \mathrm{MeV} \\
\mathrm{t}: 0^{+} \\
\text {g: } 9 / 2^{+} \\
\mathrm{m}: 1 / 2^{-}\end{array}$ & 65 & $1.83 \pm 0.22$ & {$[12]$} & $1.76 / 1.89 / 1.91 / 2.38 / 2.41 / 2.06$ & $2.10 / 2.22 / 2.16 / 2.75 / 2.76 / 2.32$ \\
\hline $\begin{array}{l}{ }^{89} Y(\gamma, 3 n)^{86} Y \\
E_{t h}=32.6 \mathrm{MeV} \\
\mathrm{t}: 1 / 2^{-} \\
\mathrm{g}: 4^{-} \\
\mathrm{m}: 8^{+}\end{array}$ & $\begin{array}{l}45 \\
55 \\
50 \\
60 \\
70\end{array}$ & $\begin{array}{c}0.199 \pm 0.026 \\
0.235 \pm 0.014 \\
0.22 \pm 0.04 \\
0.25 \pm 0.03 \\
0.26 \pm 0.03\end{array}$ & {$[14]$} & $\begin{array}{l}0.04 / 0.05 / 0.05 / 0.06 / 0.06 / 0.08 \\
0.06 / 0.07 / 0.07 / 0.09 / 0.10 / 0.13 \\
0.06 / 0.06 / 0.06 / 0.08 / 0.08 / 0.11 \\
0.06 / 0.07 / 0.07 / 0.10 / 0.11 / 0.15 \\
0.06 / 0.07 / 0.07 / 0.10 / 0.11 / 0.16\end{array}$ & $\begin{array}{l}0.05 / 0.06 / 0.05 / 0.08 / 0.08 / 0.10 \\
0.09 / 0.01 / 0.09 / 0.13 / 0.13 / 0.17 \\
0.08 / 0.08 / 0.08 / 0.11 / 0.11 / 0.14 \\
0.10 / 0.11 / 0.10 / 0.14 / 0.15 / 0.19 \\
0.12 / 0.13 / 0.12 / 0.16 / 0.17 / 0.22\end{array}$ \\
\hline $\begin{array}{l}{ }^{94} \mathrm{Mo}(\gamma, 3 n)^{91} \mathrm{Mo} \\
E_{t h}=30.4 \mathrm{MeV} \\
\text { t: } 0^{+} \\
\text {g: } 9 / 2^{+} \\
\text {m: } 1 / 2^{-}\end{array}$ & 70 & $0.62 \pm 0.06$ & {$[15]$} & $2.00 / 2.17 / 2.22 / 2.54 / 2.30 / 3.15$ & $2.48 / 2.65 / 2.60 / 3.05 / 2.76 / 3.67$ \\
\hline $\begin{array}{l}{ }^{107} \mathrm{Ag}(\gamma, 3 \mathrm{n})^{104} \mathrm{Ag} \\
E_{t h}=27.5 \mathrm{MeV} \\
\mathrm{t}: 1 / 2^{-} \\
\mathrm{g}: 5^{+} \\
\mathrm{m}: 2^{+}\end{array}$ & $\begin{array}{c}33 \\
34.5 \\
36.5 \\
38.5 \\
50\end{array}$ & $\begin{array}{c}0.88 \pm 0.14 \\
1.04 \pm 0.1 \\
1.26 \pm 0.05 \\
1.39 \pm 0.08 \\
2.33 \pm 0.22\end{array}$ & $\begin{array}{l}{[16]} \\
{[17]}\end{array}$ & $\begin{array}{l}0.34 / 0.35 / 0.35 / 0.42 / 0.47 / 0.37 \\
0.38 / 0.40 / 0.40 / 0.49 / 0.55 / 0.43 \\
0.43 / 0.45 / 0.45 / 0.57 / 0.64 / 0.49 \\
0.47 / 0.50 / 0.50 / 0.64 / 0.72 / 0.54 \\
0.57 / 0.63 / 0.64 / 0.86 / 0.97 / 0.70\end{array}$ & $\begin{array}{l}0.35 / 0.36 / 0.35 / 0.43 / 0.49 / 0.38 \\
0.39 / 0.41 / 0.41 / 0.50 / 0.57 / 0.44 \\
0.45 / 0.47 / 0.47 / 0.59 / 0.67 / 0.51 \\
0.49 / 0.52 / 0.52 / 0.67 / 0.76 / 0.57 \\
0.68 / 0.73 / 0.72 / 0.99 / 1.11 / 0.81\end{array}$ \\
\hline
\end{tabular}

Comparison isomeric ratios of different authors 


\begin{tabular}{|c|c|c|c|c|c|}
\hline \multirow[t]{2}{*}{ Reaction } & \multirow{2}{*}{$\begin{array}{l}E_{\max }, \\
\mathrm{MeV}\end{array}$} & \multirow[t]{2}{*}{$D$} & \multirow[t]{2}{*}{ Ref. } & \multicolumn{2}{|c|}{ Theoretical value of $D$} \\
\hline & & & & disabled pre-equilibrium & enabled pre-equilibrium \\
\hline $\begin{array}{l}{ }^{113} \operatorname{In}(\gamma, 3 n)^{110} \operatorname{In} \\
E_{t h}=27.1 \mathrm{MeV} \\
\mathrm{t}: 9 / 2^{+} \\
\mathrm{g}: 7^{+} \\
\mathrm{m}: 2^{+}\end{array}$ & $\begin{array}{c}32 \\
34 \\
36 \\
38.5 \\
41.5 \\
43 \\
\end{array}$ & $\begin{array}{l}0.31 \pm 0.07 \\
0.44 \pm 0.07 \\
0.62 \pm 0.06 \\
0.54 \pm 0.04 \\
0.52 \pm 0.05 \\
0.75 \pm 0.03\end{array}$ & $\begin{array}{l}{[17]} \\
{[18]}\end{array}$ & $\begin{array}{l}0.41 / 0.44 / 0.43 / 0.43 / 0.52 / 0.47 \\
0.41 / 0.44 / 0.43 / 0.44 / 0.55 / 0.51 \\
0.42 / 0.45 / 0.44 / 0.46 / 0.58 / 0.54 \\
0.43 / 0.47 / 0.46 / 0.49 / 0.61 / 0.57 \\
0.46 / 0.49 / 0.48 / 0.52 / 0.64 / 0.60 \\
0.46 / 0.50 / 0.49 / 0.54 / 0.66 / 0.61\end{array}$ & $\begin{array}{l}0.42 / 0.44 / 0.43 / 0.43 / 0.52 / 0.48 \\
0.42 / 0.44 / 0.43 / 0.44 / 0.55 / 0.51 \\
0.43 / 0.45 / 0.44 / 0.46 / 0.58 / 0.54 \\
0.44 / 0.47 / 0.46 / 0.49 / 0.61 / 0.57 \\
0.46 / 0.49 / 0.48 / 0.52 / 0.65 / 0.60 \\
0.47 / 0.50 / 0.49 / 0.54 / 0.66 / 0.61\end{array}$ \\
\hline $\begin{array}{l}{ }^{121} \mathrm{Sb}(\gamma, 3 n)^{118} \mathrm{Sb} \\
E_{t h}=25.8 \mathrm{MeV} \\
\mathrm{t}: 5 / 2^{+} \\
\mathrm{g}: 1^{+} \\
\mathrm{m}: 8^{-}\end{array}$ & $\begin{array}{l}38 \\
43\end{array}$ & $\begin{array}{l}0.14 \pm 0.04 \\
0.15 \pm 0.01\end{array}$ & {$[22]$} & $\begin{array}{l}0.05 / 0.06 / 0.05 / 0.07 / 0.08 / 0.09 \\
0.07 / 0.07 / 0.07 / 0.09 / 0.11 / 0.12\end{array}$ & $\begin{array}{l}0.06 / 0.07 / 0.06 / 0.08 / 0.09 / 0.10 \\
0.08 / 0.09 / 0.08 / 0.11 / 0.13 / 0.14\end{array}$ \\
\hline $\begin{array}{l}{ }^{140} \mathrm{Ce}(\gamma, 3 n)^{137} \mathrm{Ce} \\
E_{t h}=26.4 \mathrm{MeV} \\
\text { t: } 0^{+} \\
\text {g: } 3 / 2^{+} \\
\mathrm{m}: 11 / 2^{-}\end{array}$ & $\begin{array}{l}30 \\
70\end{array}$ & $\begin{array}{c}0.09 \pm 0.01 \\
0.9 \pm 1.0\end{array}$ & $\begin{array}{l}{[23]} \\
{[15]}\end{array}$ & $\begin{array}{l}0.09 / 0.10 / 0.09 / 0.11 / 0.11 / 0.08 \\
00.50 / 0.56 / 0.52 / 0.71 / 0.80 / 0.75\end{array}$ & $\begin{array}{l}0.10 / 0.10 / 0.09 / 0.11 / 0.11 / 0.09 \\
0.73 / 0.80 / 0.70 / 0.98 / 1.08 / 1.01\end{array}$ \\
\hline $\begin{array}{l}{ }^{144} N d(\gamma, 3 n)^{141} N d \\
E_{t h}=23.7 M e V \\
\text { t: } 0^{+} \\
\text {g: } 3 / 2^{+} \\
\text {m: } 11 / 2^{-}\end{array}$ & 70 & $0.55 \pm 0.08$ & {$[15]$} & $0.57 / 0.57 / 0.55 / 0.65 / 0.68 / 0.74$ & $0.79 / 0.79 / 0.70 / 0.88 / 0.93 / 0.98$ \\
\hline $\begin{array}{l}{ }^{165} \mathrm{Ho}(\gamma, 3 n)^{162} \mathrm{Ho} \\
E_{t h}=23.1 \mathrm{MeV} \\
\mathrm{t}: 7 / 2^{-} \\
\mathrm{g:} 1^{+} \\
\mathrm{m}: 6^{-}\end{array}$ & $\begin{array}{l}45 \\
50 \\
55 \\
60 \\
65 \\
43\end{array}$ & $\begin{array}{c}0.587 \pm 0.041 \\
0.624 \pm 0.044 \\
0.652 \pm 0.045 \\
0.637 \pm 0.045 \\
0.668 \pm 0.046 \\
1.79 \pm 0.04 \\
\end{array}$ & $\begin{array}{l}{[24]} \\
{[21]} \\
\end{array}$ & $\begin{array}{l}0.69 / 0.71 / 0.68 / 0.60 / 1.07 / 0.77 \\
0.67 / 0.71 / 0.68 / 0.60 / 1.07 / 0.77 \\
0.69 / 0.71 / 0.68 / 0.61 / 1.07 / 0.78 \\
0.69 / 0.71 / 0.68 / 0.60 / 1.07 / 0.77 \\
0.69 / 0.71 / 0.68 / 0.61 / 1.07 / 0.77 \\
0.69 / 0.70 / 0.67 / 0.60 / 1.06 / 0.77\end{array}$ & $\begin{array}{l}0.76 / 0.77 / 0.73 / 0.67 / 1.17 / 0.85 \\
0.78 / 0.79 / 0.75 / 0.69 / 1.20 / 0.88 \\
0.80 / 0.81 / 0.76 / 0.71 / 1.23 / 0.89 \\
0.81 / 0.81 / 0.76 / 0.71 / 1.23 / 0.89 \\
0.82 / 0.83 / 0.78 / 0.72 / 1.26 / 0.92 \\
0.75 / 0.76 / 0.72 / 0.66 / 1.15 / 0.85\end{array}$ \\
\hline $\begin{array}{l}{ }^{181} \mathrm{Ta}(\gamma, 3 n)^{178} \mathrm{Ta} \\
E_{t h}=22.1 \mathrm{MeV} \\
\mathrm{t}: 7 / 2^{-} \\
\mathrm{g}: 1^{+} \\
\mathrm{m}: 7^{-}\end{array}$ & $\begin{array}{l}32 \\
55\end{array}$ & $\begin{array}{c}3.0 \pm 0.6 \\
1.96 \pm 0.36\end{array}$ & $\begin{array}{l}{[25]} \\
{[26]}\end{array}$ & $\begin{array}{l}0.93 / 0.91 / 0.92 / 0.89 / 0.69 / 0.87 \\
0.80 / 0.75 / 0.77 / 0.73 / 0.54 / 0.71\end{array}$ & $\begin{array}{l}0.92 / 0.9 / 0.91 / 0.87 / 0.68 / 0.86 \\
0.70 / 0.68 / 0.7 / 0.64 / 0.48 / 0.63\end{array}$ \\
\hline $\begin{array}{l}{ }^{89} Y(\gamma, 4 n)^{85} Y \\
E_{t h}=42.2 \mathrm{MeV} \\
\mathrm{t:} 1 / 2^{-} \\
\mathrm{g}: 1 / 2^{-} \\
\mathrm{m}: 9 / 2^{+}\end{array}$ & $\begin{array}{l}60 \\
70\end{array}$ & $\begin{array}{l}0.76 \pm 0.08 \\
0.85 \pm 0.09\end{array}$ & {$[27]$} & $\begin{array}{l}1.77 / 1.86 / 1.90 / 2.49 / 3.76 / 3.36 \\
1.96 / 2.09 / 2.16 / 2.91 / 4.60 / 4.09\end{array}$ & $\begin{array}{l}1.84 / 1.92 / 1.96 / 2.57 / 3.83 / 3.43 \\
2.13 / 2.24 / 2.30 / 3.11 / 4.80 / 4.27\end{array}$ \\
\hline $\begin{array}{l}{ }^{93} \mathrm{Nb}(\gamma, 4 n)^{89} \mathrm{Nb} \\
E_{t h}=38.8 \mathrm{MeV} \\
\text { t: } 9 / 2^{+} \\
\text {g: } 9 / 2^{+} \\
\mathrm{m}: 1 / 2^{-}\end{array}$ & $\begin{array}{l}45 \\
50 \\
55 \\
60 \\
70 \\
60\end{array}$ & $\begin{array}{l}4.09 \pm 0.99 \\
5.17 \pm 0.69 \\
5.96 \pm 0.6 \\
6.6 \pm 0.9 \\
7.67 \pm 0.52 \\
6.6 \pm 0.9 \\
\end{array}$ & {$[28]$} & $\begin{array}{l}7.47 / 7.85 / 7.66 / 8.23 / 7.92 / 6.73 \\
7.91 / 8.30 / 8.18 / 8.98 / 8.87 / 8.08 \\
8.15 / 8.56 / 8.47 / 9.40 / 9.76 / 9.13 \\
8.24 / 8.65 / 8.58 / 9.57 / 10.4 / 9.73 \\
8.30 / 8.72 / 8.67 / 9.61 / 11.1 / 10.2 \\
8.24 / 8.65 / 8.58 / 9.57 / 10.4 / 9.73\end{array}$ & $\begin{array}{l}7.45 / 7.83 / 7.65 / 8.19 / 7.90 / 6.69 \\
7.86 / 8.24 / 8.14 / 8.87 / 8.84 / 7.94 \\
8.08 / 8.47 / 8.40 / 9.23 / 9.70 / 8.88 \\
8.17 / 8.55 / 8.5 / 9.38 / 10.33 / 9.42 \\
8.26 / 8.62 / 8.58 / 9.43 / 11.0 / 9.82 \\
8.17 / 8.55 / 8.5 / 9.38 / 10.33 / 9.42\end{array}$ \\
\hline $\begin{array}{l}{ }^{103} R h(\gamma, 4 n)^{99} R h \\
E_{t h}=34.7 M e V \\
\text { t: } 1 / 2^{-} \\
\text {g: } 1 / 2^{-} \\
\text {m: } 9 / 2^{+}\end{array}$ & 65 & $1.43 \pm 0.2$ & {$[30]$} & $1.54 / 1.66 / 1.76 / 2.32 / 2.18 / 1.88$ & $1.73 / 1.83 / 1.93 / 2.54 / 2.37 / 2.04$ \\
\hline
\end{tabular}




\begin{tabular}{|c|c|c|c|c|c|}
\hline \multirow[t]{2}{*}{ Reaction } & \multirow{2}{*}{$\begin{array}{l}E_{\max }, \\
\mathrm{MeV}\end{array}$} & \multirow[t]{2}{*}{$D$} & \multirow[t]{2}{*}{ Ref. } & \multicolumn{2}{|c|}{ Theoretical value of $D$} \\
\hline & & & & disabled pre-equilibrium & enabled pre-equilibrium \\
\hline $\begin{array}{l}{ }^{109} \mathrm{Ag}(\gamma, 5 n){ }^{104} \mathrm{Ag} \\
E_{t h}=44 \mathrm{MeV} \\
\mathrm{t}: 1 / 2^{-} \\
\mathrm{g}: 5^{+} \\
\mathrm{m}: 2^{+}\end{array}$ & 84 & $1.88 \pm 0.08$ & {$[19]$} & $1.32 / 1.46 / 1.46 / 2.00 / 2.22 / 1.68$ & $1.65 / 1.77 / 1.72 / 2.38 / 2.64 / 2.01$ \\
\hline $\begin{array}{l}{ }^{115} \operatorname{In}(\gamma, 5 n)^{110} \operatorname{In} \\
E_{t h}=43.4 M e V \\
\text { t: } 9 / 2^{+} \\
\text {g: } 7^{+} \\
\mathrm{m}: 2^{+}\end{array}$ & $\begin{array}{l}56 \\
75 \\
79 \\
84\end{array}$ & $\begin{array}{c}0.4 \pm 0.04 \\
0.67 \pm 0.13 \\
0.83 \pm 0.08 \\
1.02 \pm 0.02\end{array}$ & {$[19]$} & $\begin{array}{l}0.61 / 0.65 / 0.63 / 0.69 / 0.82 / 0.77 \\
0.71 / 0.75 / 0.73 / 0.83 / 0.96 / 0.88 \\
0.71 / 0.75 / 0.74 / 0.83 / 0.96 / 0.88 \\
0.71 / 0.75 / 0.74 / 0.84 / 0.97 / 0.89\end{array}$ & $\begin{array}{l}0.61 / 0.65 / 0.63 / 0.69 / 0.82 / 0.77 \\
0.72 / 0.75 / 0.73 / 0.82 / 0.94 / 0.87 \\
0.72 / 0.75 / 0.74 / 0.83 / 0.95 / 0.88 \\
0.73 / 0.76 / 0.74 / 0.83 / 0.95 / 0.89\end{array}$ \\
\hline $\begin{array}{l}{ }^{121} \mathrm{Sb}(\gamma, 5 n)^{116} \mathrm{Sb} \\
E_{t h}=43.1 \mathrm{MeV} \\
\mathrm{t}: 5 / 2^{+} \\
\mathrm{g}: 3^{+} \\
\mathrm{m}: 8^{-}\end{array}$ & 53 & $0,14 \pm 0.01$ & {$[22]$} & $0.13 / 0.14 / 0.13 / 0.18 / 0.23 / 0.11$ & $0.15 / 0.14 / 0.14 / 0.18 / 0.24 / 0.11$ \\
\hline $\begin{array}{l}{ }^{115} \operatorname{In}(\gamma, 7 n)^{108} \mathrm{In} \\
E_{t h}=61.9 \mathrm{MeV} \\
\mathrm{t}: 9 / 2^{+} \\
\mathrm{g}: 7^{+} \\
\mathrm{m}: 2^{+}\end{array}$ & 84 & $0.9 \pm 0.25$ & {$[19]$} & $1.53 / 1.61 / 1.58 / 1.65 / 2.00 / 1.72$ & $1.54 / 1.61 / 1.58 / 1.65 / 2.00 / 1.71$ \\
\hline
\end{tabular}

On Figs.1-18 we presented theoretical values of isomeric ratios (solid line corresponds to disabled preequilibrium mechanism, dotted line - to enabled) and experimental data (black triangles and red squares). We can see that close to the threshold, theoretical values of isomeric ratios calculated with and without taking into account pre-equilibrium processes are very close that means that near the threshold statistical processes dominate. Generally, at energies far from threshold, enabled pre-equilibrium mechanism gives results which better agree with experimental data (except of ${ }^{88} \mathrm{Sr}(\gamma, 3 n){ }^{85} \mathrm{Sr},{ }^{89} \mathrm{Y}(\gamma, 4 n){ }^{85} \mathrm{Y}$, $\left.{ }^{103} R h(\gamma, 4 n){ }^{99} R h,{ }^{144} N d(\gamma, 3 n){ }^{141} N d\right)$.

For some reactions we observe a good agreement between theoretical and experimental data:

$\rightarrow{ }^{55} M n(\gamma, 3 n)^{52} M n$ is well described with 1-st level density model at $49 \mathrm{MeV}$ and with 4-th, 5-th and 6-th level density models at $100 \mathrm{MeV}$;

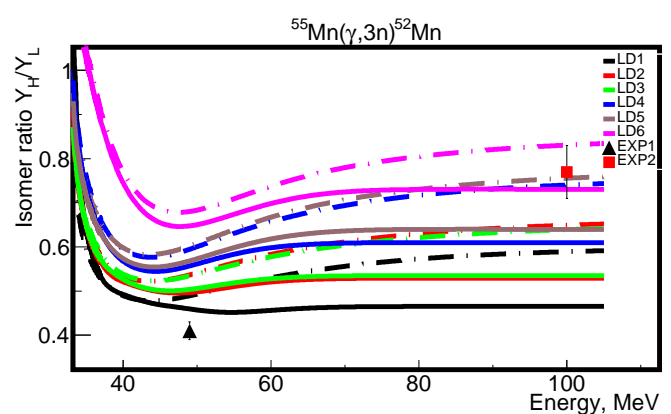

Fig. 1. ${ }^{55} M n(\gamma, 3 n)^{52} M n$. EXP1 [10], EXP2 [11] $\rightarrow{ }^{88} \mathrm{Sr}(\gamma, 3 n){ }^{85} \mathrm{Sr}$ is well-described with LD1, LD2, LD3 without pre-equilibrium mechanism;

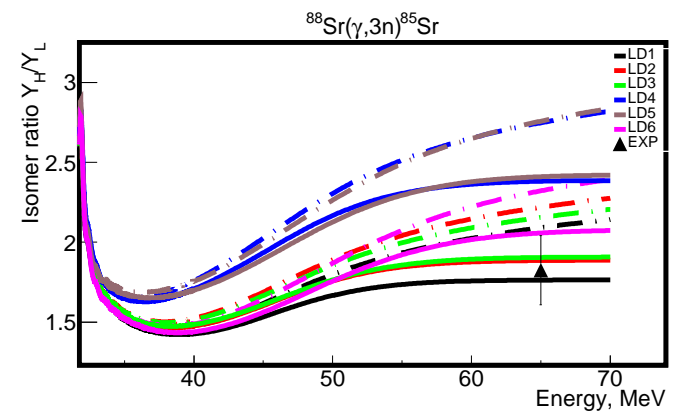

Fig.2. ${ }^{88} \operatorname{Sr}(\gamma, 3 n)^{85} \mathrm{Sr}$. EXP [12]

$\rightarrow{ }^{103} R h(\gamma, 4 n){ }^{99} R h$ is well-described with LD1 and LD2 without pre-equilibrium mechanism;

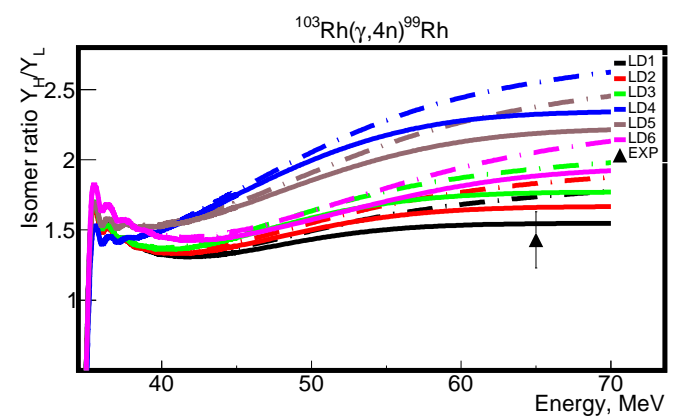

Fig. 3. ${ }^{103} R h(\gamma, 4 n){ }^{99} R h . E X P[30]$ 
$\rightarrow{ }^{109} \mathrm{Ag}(\gamma, 5 n){ }^{104} \mathrm{Ag}$ is well-described with LD4 and LD2 with pre-equilibrium mechanism;

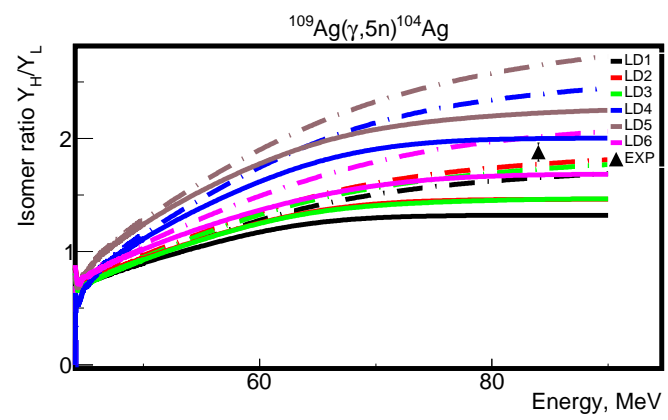

Fig.4. ${ }^{109} \mathrm{Ag}(\gamma, 5 n){ }^{104} \mathrm{Ag}$. EXP [19]

$\rightarrow$ for ${ }^{113} \operatorname{In}(\gamma, 3 n)^{110}$ In the difference between theoretical models is of one order with statistical error of experimental data, and all theoretical models are in good agreement with experiment;

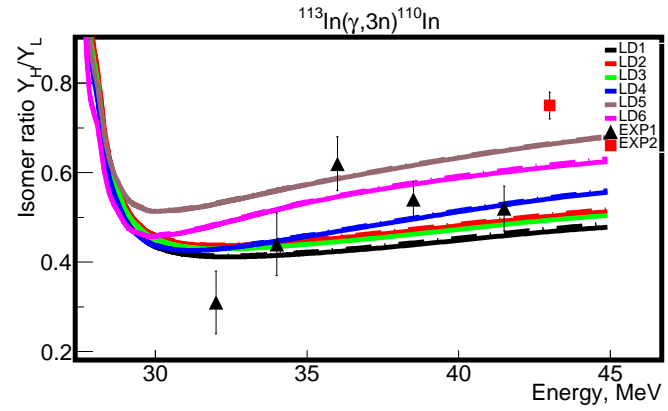

Fig. 5. ${ }^{113} \operatorname{In}(\gamma, 3 n)^{110}$ In. EXP [21]

$\rightarrow{ }^{121} S b(\gamma, 5 n){ }^{116} S b$ is well-described with LD2 and LD3;

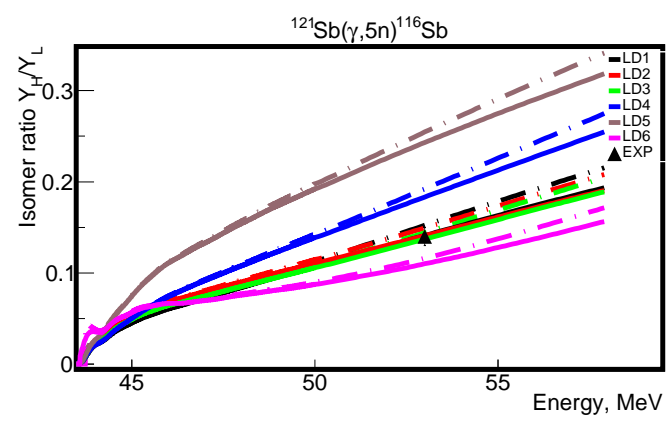

Fig. 6. ${ }^{121} S b(\gamma, 5 n){ }^{116} S b$. EXP [22]

$\rightarrow{ }^{121} \mathrm{Sb}(\gamma, 3 n){ }^{118} \mathrm{Sb}$ is well-described with LD5 and LD6 with enabled pre-equilibrium mechanism;

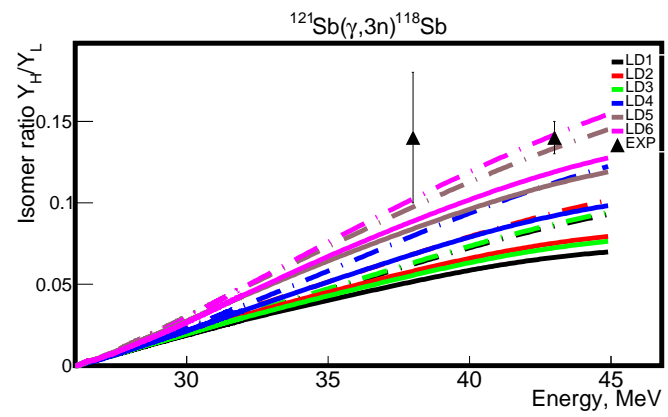

Fig. 7. ${ }^{121} S b(\gamma, 3 n){ }^{118} S b$. EXP [22]

$\rightarrow{ }^{140} \mathrm{Ce}(\gamma, 3 n){ }^{137} \mathrm{Ce}$ is well-described with LD1 at $30 \mathrm{MeV}$; and $70 \mathrm{MeV}$ statistical error of experimental data covers all theoretical models;

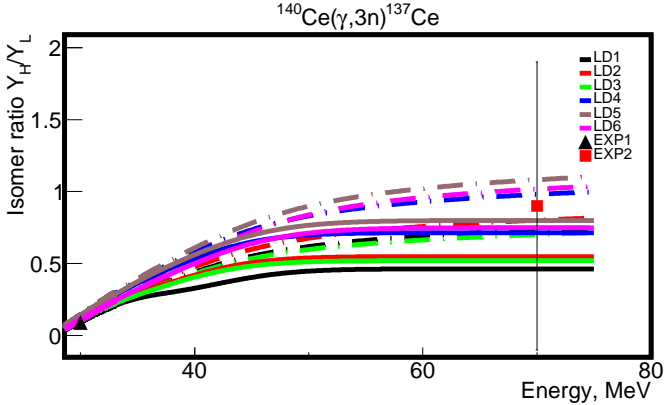

Fig.8. ${ }^{140} \mathrm{Ce}(\gamma, 3 n){ }^{137} \mathrm{Ce} . \mathrm{EXP1}$ [23], EXP2 [15]

$\rightarrow{ }^{144} \mathrm{Nd}(\gamma, 3 n)^{141} \mathrm{Nd}$ is well-described with LD1, LD2, LD3 and LD4;

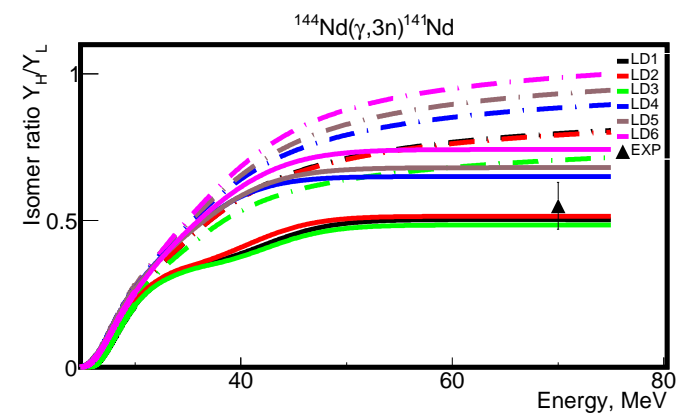

Fig. 9. ${ }^{144} N d(\gamma, 3 n){ }^{141} N d$. EXP [15]

$\rightarrow{ }^{165} \mathrm{Ho}(\gamma, 3 n){ }^{162} \mathrm{Ho}$ : experimental data (except of [21]) are well-described with LD4.

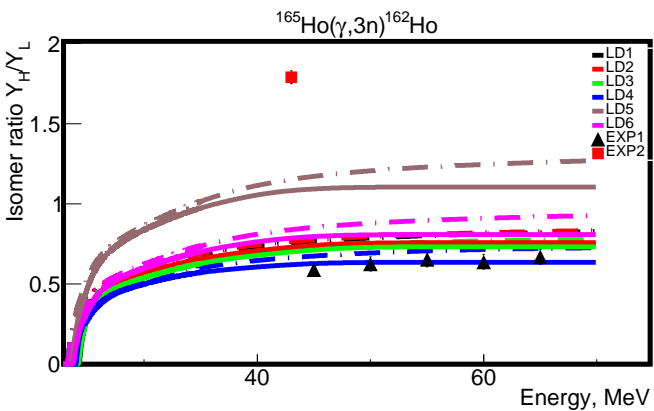

Fig. 10. ${ }^{165} \mathrm{Ho}(\gamma, 3 n)^{162} \mathrm{Ho}$. EXP1 [24], EXP2 [21]

There are reactions with significant differences between experimental data and theoretical predictions:

$\rightarrow{ }^{89} Y(\gamma, 4 n){ }^{85} Y$ and ${ }^{89} Y(\gamma, 3 n)^{86} Y$. In one case all theoretical models give overestimated values; and in the other case, in opposite, underestimated. It should be noted that for ${ }^{89} Y(\gamma, 3 n){ }^{86} Y$ experimental data measured by different groups at different experiments are consistent among themselves;

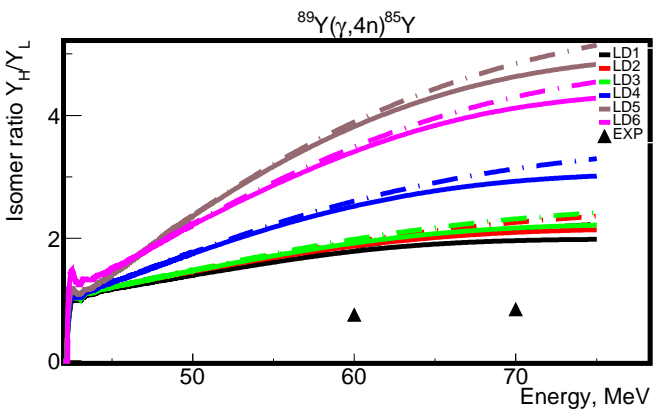

Fig. 11. ${ }^{89} Y(\gamma, 4 n){ }^{85} Y . \operatorname{EXP}[27]$ 


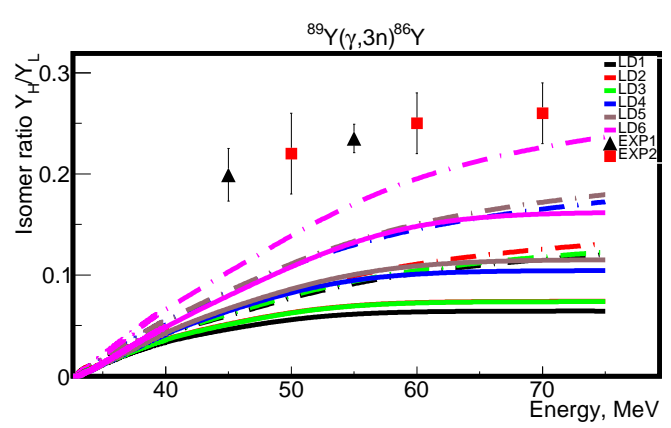

Fig. 12. ${ }^{89} Y(\gamma, 3 n){ }^{86} Y$. EXP1 [13], EXP2 [14]

$\rightarrow{ }^{93} \mathrm{Nb}(\gamma, 4 n){ }^{89} \mathrm{Nb}$ : all theoretical models overestimate isomeric ratios, but it should be noted that LD1 describes well the behavior of isomeric ratio energy dependence;

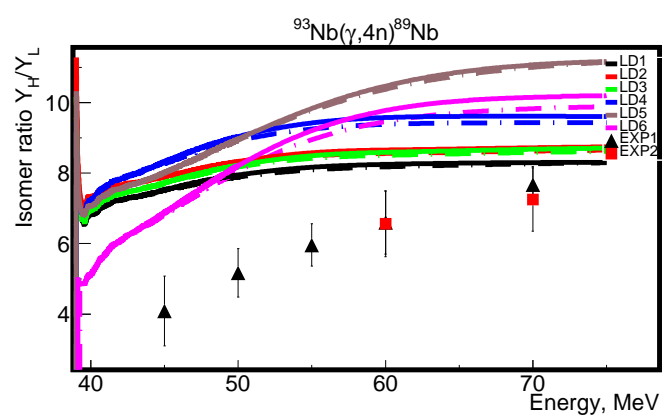

Fig. 13. ${ }^{93} N b(\gamma, 4 n){ }^{89} N b$. EXP1 [28], EXP2 [29] $\rightarrow{ }^{94} M o(\gamma, 3 n)^{91} M o$ : all theoretical models give overvalued results;

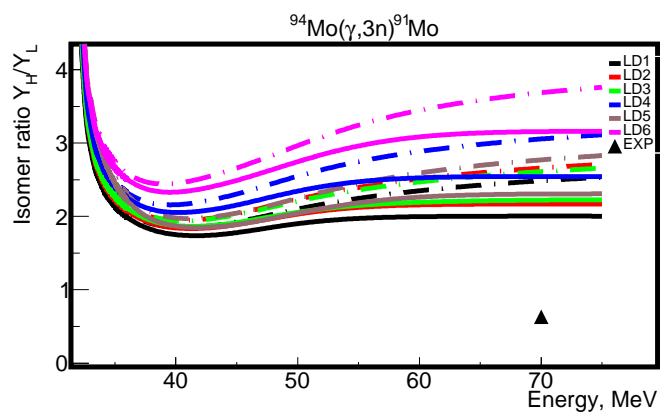

Fig. 14. ${ }^{94} M o(\gamma, 3 n){ }^{91} M o . \operatorname{EXP}[15]$

$\rightarrow{ }^{107} \mathrm{Ag}(\gamma, 3 n){ }^{104} \mathrm{Ag}$ : all theoretical models underestimate isomeric ratios. It should be noted that experimental data measured by different groups at different experiments are consistent among themselves;

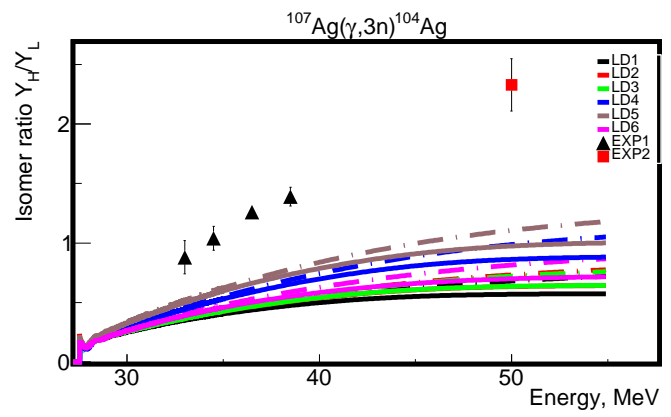

Fig. 15. ${ }^{107} \mathrm{Ag}(\gamma, 3 n){ }^{104} \mathrm{Ag}$. EXP1 [16,17], EXP2 [20]

$\rightarrow{ }^{115} \operatorname{In}(\gamma, 7 n)^{108}$ In: all theoretical models give overvalued results;

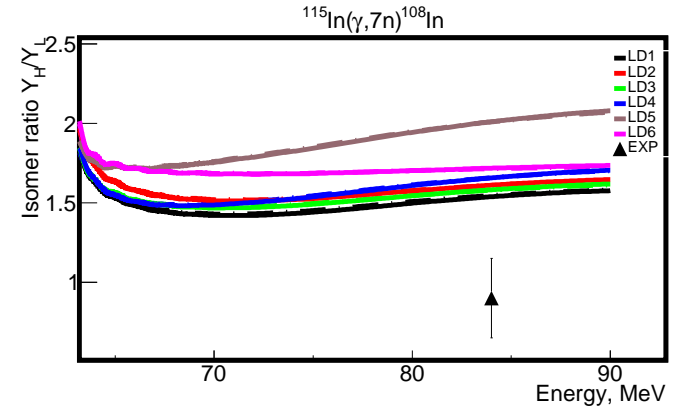

Fig. 16. ${ }^{115} \operatorname{In}(\gamma, 7 n)^{108}$ In. EXP [19]

$\rightarrow{ }^{115} \operatorname{In}(\gamma, 5 n){ }^{110}$ In: all theoretical models give values of one order with experimental data, but they do not predict the behavior of isomeric ratios energy dependence;

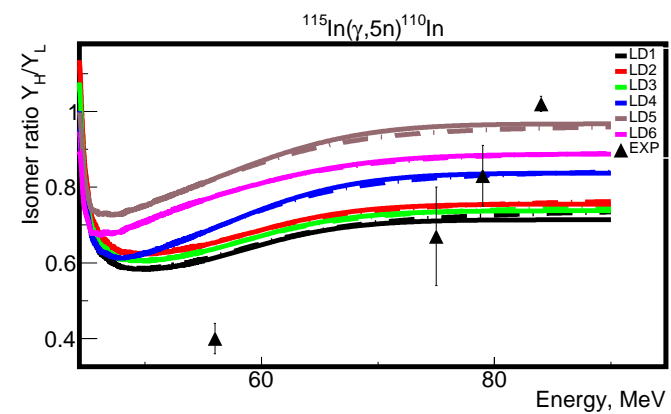

Fig.17. ${ }^{115} \operatorname{In}(\gamma, 5 n){ }^{110}$ In. EXP [19] $\rightarrow{ }^{181} \mathrm{Ta}(\gamma, 3 \mathrm{n})^{178} \mathrm{Ta}$ : at $30 \mathrm{MeV}$ theoretical models underestimate isomeric ratio, and at $55 \mathrm{MeV}$, in opposite, theory gives overvalued results.

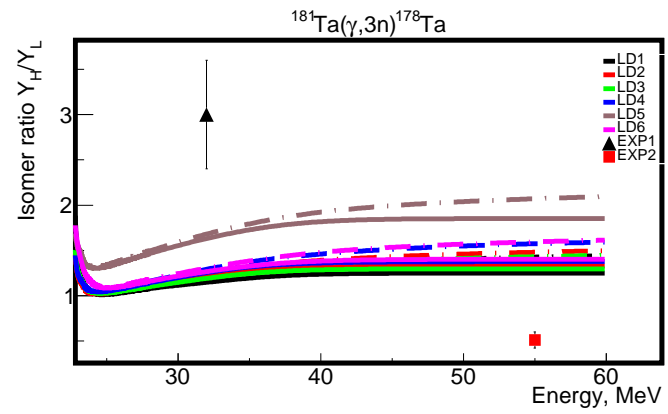

Fig. 18. ${ }^{181} \mathrm{Ta}(\gamma, 3 n)^{178} \mathrm{Ta}$. EXP1 [25], EXP2 [26]

\section{CONCLUSIONS}

In this work isomeric ratios of photonuclear reactions, measured by different research groups were collected, systemized and compared to theoretical predictions. For some reactions theoretical values are in good agreement with experiment, for some of them we observe significant difference. As a general conclusion from the analysis of the results obtained, we can assume that a shortage of experimental data significantly limits the possibilities of choice between different theoretical models and approaches. Overview provided in this work will facilitate search of the most reliable data, the work on estimation of available data and planning of new data obtaining.

\section{Acknowledgments}

Research was conducted in the scope of the IDEATE International Associated Laboratory (LIA). The 
TSNUK contribution was partly supported by the State Fund for Fundamental Researches of Ukraine (project F79/-2018 in the frame of LIA IDEATE developments, Research Grant 2-ND/18, Research Grant 4-N-2018).

\section{References}

1. www.talys.eu

2. R. Capote, et al. RIPL - Reference Input Parameter Library for calculation of nuclear reactions and nuclear data evaluation // Nucl. Data Sheets. 2009, v.110, p.3107.

3. A. Gilbert, A.G.W. Cameron. A composite nuclear-level density formula with shell corrections // Canadian Journal of Physics. 1965, v.43, p.1446.

4. W. Dilg, W.Schantl, H. Vonach, M. Uhl. Level density parameters for the back-shifted fermi gas model in the mass range $40<A<250 / / N u$ clear Physics. 1973, v.A217, p.269.

5. A.V. Ignatyuk, K.K. Istekov, G.N. Smirenkin. Collective effects in level density, and the probability of fission // Soviet Journal of Nuclear Physics. 1979, v.29, N4, p.450.

6. A.V. Ignatyuk, J.L. Weil, S. Raman, S. Kahane. Density of discrete levels in ${ }^{116} \mathrm{Sn} / /$ Physical Review. 1993, v.C47, p.1504.

7. V.G. Nedorezov, Yu.N. Ranyuk. Photofission of nuclei behind giant resonance. Kiev: "Naukova Dumka", 1989 (in Ukrainian).

8. L.I. Schiff. Energy-angle distribution of thin target bremsstrahlung // Phys. Rev. 1951, v.83, p.252-253.

9. B.S. Ishkhanov, I.M. Kapitonov. Interaction of electromagnetic radiation with atomic nuclei. Moscow: Publishing house of the Moscow University, 1979 (in Russian).

10. R. Volpel. Bestimmung von Isomerenverhiiztnissen bei Kernphoto-und (n,2n)-Reaktionen // Nuclear Physics. 1972, v.A182, N2, p.411-416.

11. W.B. Walters, J.P. Hummel. Studies of Isomeric Yield Ratios in the Production of ${ }^{44} S c,{ }^{52} \mathrm{Mn}$ and ${ }^{87} Y$ by Photonuclear Reactions // Physical Review. 1966, v.150, N3, p.867-876.

12. Tran Duc Thiep, et al. The isomeric ratios in photonuclear reactions of natural strontium induced by bremsstrahlungs in the giant dipole resonance region and by $65 \mathrm{MeV}$ bremsstrahlung // $R a$ dioanalytical and nuclear chemistry. 2010, v.286, p.161-167.
13. Mansoureh Tatari, et al. Measurement of isomeric yield ratios of ${ }^{86 m, g} \mathrm{Y},{ }^{87 m, g} \mathrm{Y}$, and ${ }^{89 m, g} \mathrm{Zr}$ in the photonuclear reactions of ${ }^{89} Y$ and ${ }^{n a t} Z r$ at the end-point bremsstrahlung // Nuclear Instruments and Methods in Physics Research. 2015, v.B244, p.76-82.

14. Md. Shakilur Rahman, et al. Measurement of isomeric yield ratios for ${ }^{90} Z r(\gamma, n)^{89 m, g} Z r, \quad{ }^{n a t} Z r(\gamma, x n 1 p)^{86 m, g} Y$, and ${ }^{89} Y(\gamma, x n)^{87 m, g, 86 m, g} Y$ reactions with $50-$, $60-$, and $70-\mathrm{MeV}$ bremsstrahlung // Nuclear Instruments and Methods in Physics Research. 2009, v.B267, p.3511-3518.

15. P.E. Haustein, A.F. Voigt. Isomer ratio measurements for ${ }^{91} \mathrm{Mo},{ }^{137} \mathrm{Ce}$ and ${ }^{144} \mathrm{Nd}$ produced by $(\gamma, n)$ and $(\gamma, 3 n)$ reactions // Inorganic Nuclear Chemistry. 1971, v.33, p.289-294.

16. O.A. Bezshyyko, A.N. Vodin , L.O. GolinkaBezshyyko, A.N. Dovbnya, et al. Isomer ratios for product of photonuclear reaction ${ }^{107} \mathrm{Ag}(\gamma, 3 n)^{104 m, g} \mathrm{Ag}$ in the energy region $35 . .40 \mathrm{MeV} / /$ Nuclear Physics and Atomic Energy. 2009, v.10, p.61-65.

17. O.A. Bezshyyko, A.N. Vodin , L.O. GolinkaBezshyyko, A.N. Dovbnya, et al. Isomer ratios of products from photonuclear reactions on silver and indium nuclei at $\gamma$-ray energies above $35 \mathrm{MeV}$ // Bulletin of the Russian Academy of Sciences: Physics. 2009, v.73, p.1461-1465.

18. O.A. Bezshyyko, A.N. Vodin , L.O. GolinkaBezshyyko, A.N. Dovbnya, et al. Isomer ratios of photonuclear reaction products on silver and indium isotopes for bremsstrahlung energies above $35 \mathrm{MeV}$. 2nd // International Conference on Current Problems in Nuclear Physics and Atomic Energy. NPAE 2008 - Proceedings, 2009, p.252-256.

19. O.A. Bezshyyko, A.N. Vodin, L.O. GolinkaBezshyyko, A.M. Dovbnya, et al. Isomer Ratios for Products of Photonuclear Reactions with Middle-Weight Nuclei // Bulletin of the Russian Academy of Sciences. Physics. 2011, v.75, N7, p.941-945.

20. A. Ermakov, et al. Excitation of $2+$ isomeric level of ${ }^{104} \mathrm{Ag}$ nucleus in photonuclear reactions // Conf. on Nucl. Data for Sci. and Technology. Nice, 2007.

21. D. Kolev, et al. A convenient method for experimental determination of yields and isomeric ratios in photonuclear reactions measured by the activation technique // Nuclear Instruments and Methods in Physics Research. 1995, v.A356, p.390-396.

22. O. Bezshyyko, A. Dovbnya, L. GolinkaBezshyyko, I. Kadenko, et al. Isomer ratios 
for products of photonuclear reactions on ${ }^{121} \mathrm{Sb} / /$ European Physics Journal Web of Conferences. 2017, v.146, p.05016.

23. J.H. Carver, G.E. Coote, T.R. Sherwood. Isomeric $(\gamma, \mathrm{n})$ cross section ratios and the spin dependence of the nuclear level density // Nuclear Physics. 1962, v.37, p.449-456.

24. V.D. Nguyen, et al. Measurement of isomeric yield ratios for the ${ }^{n a t} \mathrm{Ho}(\gamma, x n)^{164 m, g ; 162 m, g} \mathrm{Ho}$ reactions in the bremsstrahlung energy region from 45 to $65 \mathrm{MeV} / /$ Journal of Radioanalytical and Nuclear Chemistry. 2013, v.298, p.1447-1452.

25. J.H. Carver, W. Turchinetz. The $(\gamma, 2 \mathrm{n})$ and $(\gamma, 3 \mathrm{n})$ Reactions in ${ }^{181} \mathrm{Ta} / /$ Proceedings of the Physical Society. 1958, v.A71, p.613-617.

26. H. Bartsch, K. Huber, U. Kneissl, H. Krieger. Critical consideration of the statistical model analysis of photonuclear isomeric cross-section ratios // Nuclear Physics. 1976, v.A256, p.243252 .
27. M.S. Rahman, et al. Measurements of Isomeric Yield Ratios of ${ }^{44 m, g} S c$ and ${ }^{85 m, g} Y$ with Intermediate Energy Bremsstrahlung // Journal of the Korean Physical Society. 2011, v.59, N2, p.17491752.

28. H. Naik, et al. Independent isomeric-yield ratio of ${ }^{89 m, g} \mathrm{Nb}$ from ${ }^{93} \mathrm{Nb}(\gamma, 4 n)$, ${ }^{\text {nat }} \mathrm{Zr}(p, x n)$, and ${ }^{89} Y(\gamma, 4 n)$ reactions // Journal of Radioanalytical and Nuclear Chemistry. 2014, v.299, p.13351343.

29. K.S. Kim, et al. Measurement of isomeric yield ratios for ${ }^{93} N b(\gamma, 4 n)^{89 m, g} N b$ and ${ }^{n a t} \mathrm{Mo}(\gamma, x n 1 p)^{95 m, g} \mathrm{Nb}$ reactions with 50-, 60-, and 70-MeV bremsstrahlung // Journal of Radioanalytical and Nuclear Chemistry. 2011, v.287, p.867-877.

30. V.D. Nguyen, et al. Measurement of Isomeric Cross-Section Ratios for the ${ }^{45} S c(\gamma, n){ }^{44 m, g} S c$, ${ }^{n a t} \mathrm{Ti}(\gamma, x){ }^{44 m, g} \mathrm{Sc}, \quad{ }^{103} \mathrm{Rh}(\gamma, 4 n){ }^{99 m, g} \mathrm{Rh}, \quad$ and ${ }^{n a t} \mathrm{Fe}(\gamma, x)^{52 m, g} \mathrm{Mn} / /$ Journal of the Korean Physical Society. 2007, v.50, N2, p.417-425.

\title{
ИЗОМЕРНЫЕ ОТНОШЕНИЯ В ФОТОЯДЕРНЫХ РЕАКЦИЯХ С МНОЖЕСТВЕННОЙ ЭМИССИЕЙ НЕЙТРОНОВ
}

\author{
А. Н. Водин, О.А.Безшейко, Л. А. Голинка-Безшейко, И. Н. Каденко, В.А. Кушнир, \\ А.В. Котенко, А.В. Лубинети, В.В. Митроченко, С.Н. Олейник, С. А. Пережогин, \\ C. Vallerand
}

Целью работы является изучение зависимости изомерных отношений в реакциях $A(\gamma, x n)^{m, g}(A-x n)$ от энергии гамма-квантов в диапазоне $35 \ldots 100$ МэВ, т. е. за пределами области гигантского дипольного резонанса (ГДР). Экспериментальные данные были взяты из международной базы данных EXFOR для широкого спектра ядерных масс $(55<A<181)$. Теоретические значения изомерных соотношений получены с использованием кода TALYS-1,8. В наших симуляциях были рассмотрены несколько моделей плотности уровней с учетом предравновесных механизмов. Полученные результаты позволяют сделать выводы о различных механизмах фотоядерных реакций на некоторых ядрах и об их относительном вкладе в зависимости от энергии $\gamma$-квантов.

\section{ІЗОМЕРНІ ВІДНОШЕННЯ У ФОТОЯДЕРНИХ РЕАКЦІЯХ З МНОЖИННОЮ ЕМІСІЄЮ НЕЙТРОНІВ}

О. М.Водін, О.А.Безшейко, Л. О. Голінка-Безшейко, І. М. Каденко, В.А. Кушнір, А.В.Котенко, О.В.Лубинети, В.В.Мітроченко, С.М. Олійник, С.А.Пережогин, C. Vallerand

Метою роботи є вивчення залежності ізомерних відношень у реакціях $A(\gamma, x n)^{m, g}(A-x n)$ від енергії гамма-квантів у діапазоні $35 \ldots 100 \mathrm{MeB}$, тобто за межами області гігантського дипольного резонансу (ГДР). Експериментальні дані були взяті з міжнародної бази даних EXFOR для широкого спектра ядерних мас $(55<A<181)$. Теоретичні значення ізомерних співвідношень отримані з використанням коду TALYS-1,8. У наших симуляціях були розглянуті кілька моделей щільності рівнів з урахуванням передрівноважних механізмів. Отримані результати дозволяють зробити висновки про різні механізми фотоядерних реакцій на деяких ядрах і про їх відносний вклад у залежності від енергії $\gamma$-квантів. 